\title{
RESEARCH
}

Open Access

\section{Effects of iron chelation therapy on the clinical course of aceruloplasminemia: an analysis of aggregated case reports}

\author{
Lena H. P. Vroegindeweij ${ }^{1}$, Agnita J. W. Boon ${ }^{2}$, J. H. Paul Wilson ${ }^{1}$ and Janneke G. Langendonk ${ }^{1 *}$
}

\begin{abstract}
Background: Aceruloplasminemia is a rare genetic iron overload disorder, characterized by progressive neurological manifestations. The effects of iron chelation on neurological outcomes have only been described in case studies, and are inconsistent. Aggregated case reports were analyzed to help delineate the disease-modifying potential of treatment.
\end{abstract}

Methods: Data on clinical manifestations, treatment and neurological outcomes of treatment were collected from three neurologically symptomatic Dutch patients, who received deferiprone with phlebotomy as a new therapeutic approach, and combined with other published cases. Neurological outcomes of treatment were compared between patients starting treatment when neurologically symptomatic and patients without neurological manifestations.

Results: Therapeutic approaches for aceruloplasminemia have been described in 48 patients worldwide, including our three patients. Initiation of treatment in a presymptomatic stage of the disease delayed the estimated onset of neurological manifestations by 10 years (median age 61 years, SE 5.0 vs. median age 51 years, SE 0.6, $p=0.001$ ). Although in 11/20 neurologically symptomatic patients neurological manifestations remained stable or improved during treatment, these patients were treated significantly shorter than patients who deteriorated neurologically (median 6 months vs. median 43 months, $p=0.016$ ). Combined iron chelation therapy with deferiprone and phlebotomy for up to 34 months could be safely performed in our patients without symptomatic anemia (2/3), but did not prevent further neurological deterioration.

Conclusions: Early initiation of iron chelation therapy seems to postpone the onset of neurological manifestations in aceruloplasminemia. Publication bias and significant differences in duration of treatment should be considered when interpreting reported treatment outcomes in neurologically symptomatic patients. Based on theoretical grounds and the observed long-term safety and tolerability in our study, we recommend iron chelation therapy with deferiprone in combination with phlebotomy for aceruloplasminemia patients without symptomatic anemia.

Keywords: Aceruloplasminemia, Neurological manifestations, Iron chelation therapy, Deferiprone, Phlebotomy

\footnotetext{
* Correspondence: j.langendonk@erasmusmc.nl

'Department of Internal Medicine, Center for Lysosomal and Metabolic

Diseases, Porphyria Center Rotterdam, Erasmus MC University Medical Center Rotterdam, Rotterdam, The Netherlands

Full list of author information is available at the end of the article
}

C C The Author(s). 2020 Open Access This article is licensed under a Creative Commons Attribution 4.0 International License, which permits use, sharing, adaptation, distribution and reproduction in any medium or format, as long as you give appropriate credit to the original author(s) and the source, provide a link to the Creative Commons licence, and indicate if changes were made. The images or other third party material in this article are included in the article's Creative Commons licence, unless indicated otherwise in a credit line to the material. If material is not included in the article's Creative Commons licence and your intended use is not permitted by statutory regulation or exceeds the permitted use, you will need to obtain permission directly from the copyright holder. To view a copy of this licence, visit http://creativecommons.org/licenses/by/4.0/. The Creative Commons Public Domain Dedication waiver (http://creativecommons.org/publicdomain/zero/1.0/) applies to the data made available in this article, unless otherwise stated in a credit line to the data. 


\section{Background}

Aceruloplasminemia (OMIM \#604290) is a rare form of neurodegeneration with brain iron accumulation (NBIA), characterized by progressive neurological deterioration and iron accumulation in visceral organs [1]. Iron chelation therapy has been shown by biochemical markers and quantitative visceral MRI to reduce body iron stores, but the reported effects on neurological outcomes are inconsistent $[2,3]$.

Iron accumulation in aceruloplasminemia is caused by a lack of ceruloplasmin ferroxidase activity. The failure to convert ferrous iron $\left(\mathrm{Fe}^{2+}\right)$ to ferric iron $\left(\mathrm{Fe}^{3+}\right)$ at the cell surface results in low transferrin saturation $[1,4,5]$. Deferiprone is the only chelator available capable of transporting iron across cell membranes and across the blood-brain barrier. At low transferrin saturation, deferiprone additionally mediates shuttling of iron from excess iron stores to erythroid cells by transferring iron to transferrin [6]. However, deferiprone monotherapy is not highly effective in reducing body iron stores in aceruloplasminemia [7, 8], and needs to be combined with another chelator to achieve satisfactory rates of iron removal [8-10]. The deferiprone-induced redistribution of iron to hematopoietic tissues might permit a combination with phlebotomy as a new therapeutic approach for aceruloplasminemia. Deferiprone with phlebotomy has the potential to reduce both cerebral and systemic iron stores more rapidly than previously achieved, and possibly to slow disease progression.

Due to the rarity of aceruloplasminemia, randomized controlled study designs are not feasible to explore clinical outcomes of iron chelation therapy. The value of aggregated case reports has recently been highlighted as an alternative means of systematically deriving evidence from the literature in rare diseases $[11,12]$. However, the rich source of information embedded in published case reports and case series has not yet been quantitatively investigated in such manner for aceruloplasminemia.

We performed an analysis of aggregated case reports of aceruloplasminemia and present detailed clinical outcomes of long-term treatment with deferiprone and phlebotomy in three Dutch patients. The aim of our study was to increase knowledge of the natural history of aceruloplasminemia and disease-modifying potential of iron chelation therapy.

\section{Methods}

\section{Subjects}

Three Dutch patients with homozygosity for G631R in the ceruloplasmin $(C P)$ gene were enrolled in the study: two brothers with neurological symptoms and their first cousin with minimal neurological complaints. The neurological presentation of all patients has been previously published [13-16]. The study was approved by the
Medical Ethics Review Board of the Erasmus MC. All participants gave written informed consent.

\section{Procedures}

Deferiprone was combined with phlebotomy in all patients. Deferiprone starting dose was $15 \mathrm{mg} / \mathrm{kg} /$ day given orally in two doses, followed by titration to a maximum dose of $65 \mathrm{mg} / \mathrm{kg} /$ day. Phlebotomy was started by withdrawing $300 \mathrm{ml}$ blood every fortnight and subsequently increased to $500 \mathrm{ml}$ every 3 weeks.

Safety and tolerability of deferiprone were strictly monitored, as deferiprone is rarely associated with neutropenia (neutrophil count $<1.5 \cdot 10^{9} / \mathrm{l}$ ) and agranulocytosis (neutrophil count $<0.5 \cdot 10^{9} / 1$ ). Neutrophils were measured weekly during deferiprone titration and every 2 weeks after the maximum dose of deferiprone had been reached. In patients who developed neutropenia, the dose of deferiprone was halved and neutrophil counts were monitored weekly. If neutrophil counts recovered, deferiprone was gradually increased to its original dosage. If neutrophils remained below the lower limit, deferiprone was stopped and not reintroduced. Transaminases (AST, ALT) were measured monthly and zinc levels every 6 months. Hemoglobin levels were measured prior to each phlebotomy. Phlebotomy was postponed if the hemoglobin level was more than $3.25 \mathrm{~g} / \mathrm{dl}$ lower $(2 \mathrm{mmol} / \mathrm{l})$ than at baseline or the patient mentioned complaints associated with anemia.

Effectiveness was assessed every 3 months during the first year, followed by half-year evaluations in the following years of treatment. Neurological function was evaluated and videotaped by a movement disorders specialist (A.B.). The Unified Parkinson's Disease Rating Scale (UPDRS/III) was used to measure motor symptoms of parkinsonism and the Scale for the Assessment and Rating of Ataxia (SARA) for the assessment of coordination in several tasks including speech and gait. The Hospital Anxiety and Depression Scale (HADS) evaluated levels of anxiety and depression and was used as a self-assessment questionnaire. Ophthalmic examinations for the assessment of retinopathy were performed at yearly intervals. Iron depositions in the brain were qualitatively assessed by yearly T2 and T2\%-weighted MRI, as hypo-intensities on these sequences are indicative of iron accumulation. Images were acquired using an individualized protocol at either $1.5 \mathrm{~T}$ or $3 \mathrm{~T}$, depending on the magnetic field strength of the first MRI, and evaluated by a neuroradiologist. Quantitative comparisons could not be performed. Systemic iron stores were followed by serum ferritin and HbA1c levels. Serum ferritin, reflecting iron stores in the liver, was determined together with CRP, AST and ALT, to permit interpretation of changes in serum ferritin values. HbA1c was used as a marker of glycemic control 
and development of diabetes following pancreatic iron accumulation.

\section{Treatment parameters}

The primary endpoints were safety and change from baseline in UPDRS/III and SARA scores. Changes in HADS scores, qualitative assessment of brain iron as evaluated by MRI, and changes in ferritin and HbA1c values served as secondary effectiveness endpoints.

\section{Review of the literature}

Case reports and case series that described treatment for aceruloplasminemia were collected by updating a previously published literature search till December 2019 [13]. Gender, ethnicity, age at first manifestations, age at onset of neurological manifestations, age at initiation of treatment, duration of treatment, neurological outcomes of treatment and age at death were extracted from all identified case studies. The authors of these cases were asked for additional follow-up of the patients.

\section{Statistics}

Ages and duration of treatment were presented as median (interquartile range) values. Differences in gender, ethnicity, age at first manifestations, age at initiation of treatment and duration of treatment between patients with neurological manifestations at the start of treatment and neurologically asymptomatic patients were explored using the Mann-Whitney U-test or $\chi^{2}$ test, where appropriate. Differences in the clinical course of the disease and onset of neurological manifestations were explored using Kaplan-Meier analysis. Patients who remained neurologically asymptomatic were censored at the age at end of follow-up, which was obtained from either later published articles or direct contact with the authors. If additional follow-up was not available, the age at end of follow-up was calculated using the reported age at initiation of treatment and duration of treatment. Statistical significance was defined as $p<0.05$. All analyses were performed using SPSS 25 (IBM, Armonk, NY).

\section{Results \\ Deferiprone with phlebotomy in three G631R homozygous patients}

At the time of enrolment, both brothers (case 1,2) had a two-year history of neurological manifestations and had been treated with phlebotomy monotherapy for several months, which resulted in mildly reduced hemoglobin levels. Both presented initially with normal hemoglobin levels. Their first cousin (case 3) reported stable neurological disease after 13 years of deferoxamine treatment (1000 mg/day, s.c., twice weekly) [16]. He was known with microcytic anemia since childhood, which had been treated intermittently with oral iron supplements. At the time of counselling, microcytic anemia was considered to be the first manifestation of aceruloplasminemia in case 3 , following routine exclusion of other causes.

The combination of deferiprone and phlebotomy was administered for 15 months in case 1, 34 months in case 2 and 1 month in case 3 . Total patient follow-up ranged from 18 to 76 months. Premature discontinuation of treatment was due to neurological deterioration in case 1 and 2, in whom the combination of deferiprone and phlebotomy was generally well tolerated. In case 3 , phlebotomy was discontinued due to anemia-related fatigue and dyspnea following two venesections of $300 \mathrm{ml}$. He was subsequently treated by deferiprone in combination with deferoxamine (1000 mg/day s.c., once-twice weekly). A detailed overview of the biochemical sequelae and tolerance of iron chelation therapy in our three cases can be obtained from Additional file 1 .

Table 1 summarizes the clinical outcomes of treatment with deferiprone and phlebotomy (case 1,2) and deferiprone in combination with deferoxamine (case 3). All patients deteriorated neurologically, as illustrated by the gradual increase in UPDRS/III and SARA scores over time (Fig. 1). Patient ratings of anxious and depressive feelings

Table 1 Clinical characteristics of our G631R homozygous patients at baseline and end of follow-up

\begin{tabular}{|c|c|c|c|c|c|c|c|c|c|}
\hline \multirow{2}{*}{$\begin{array}{l}\text { Case/gender/ } \\
\text { age }^{a}\end{array}$} & \multirow{2}{*}{$\begin{array}{l}\text { Follow-up } \\
\text { (months) }\end{array}$} & \multicolumn{2}{|l|}{ Neurological function } & \multicolumn{2}{|c|}{ Retinopathy } & \multicolumn{2}{|l|}{ Diabetes } & \multicolumn{2}{|l|}{ Anemia } \\
\hline & & Baseline & $\mathrm{EFU}$ & Baseline & EFU & Baseline & $\mathrm{EFU}$ & Baseline & EFU \\
\hline $1 / \mathrm{M} / 50$ & 18 & $\begin{array}{l}\text { Orofacial dyskinesia, chorea, } \\
\text { dystonia, dysarthria, gait } \\
\text { disturbance }\end{array}$ & Died & No & NA & No & Yes & Yes $^{b}$ & Yes \\
\hline $2 / M / 56$ & 76 & $\begin{array}{l}\text { Dysarthria, ataxia, gait } \\
\text { disturbance, behavioral } \\
\text { changes }\end{array}$ & $\begin{array}{l}\text { Progressive speech and } \\
\text { gait disturbance, } \\
\text { wheelchair-bound, aspiration, } \\
\text { cognitive decline and apathy }\end{array}$ & No & No & Yes & Yes & $Y_{e s}^{b}$ & No \\
\hline 3/M/61 & 70 & $\begin{array}{l}\text { Stress related tremor, slightly } \\
\text { diminished facial expression }\end{array}$ & $\begin{array}{l}\text { Development of gait } \\
\text { disturbance with falls, mental } \\
\text { slowing, behavioral changes }\end{array}$ & No & No & No & Yes & Yes & Yes \\
\hline
\end{tabular}




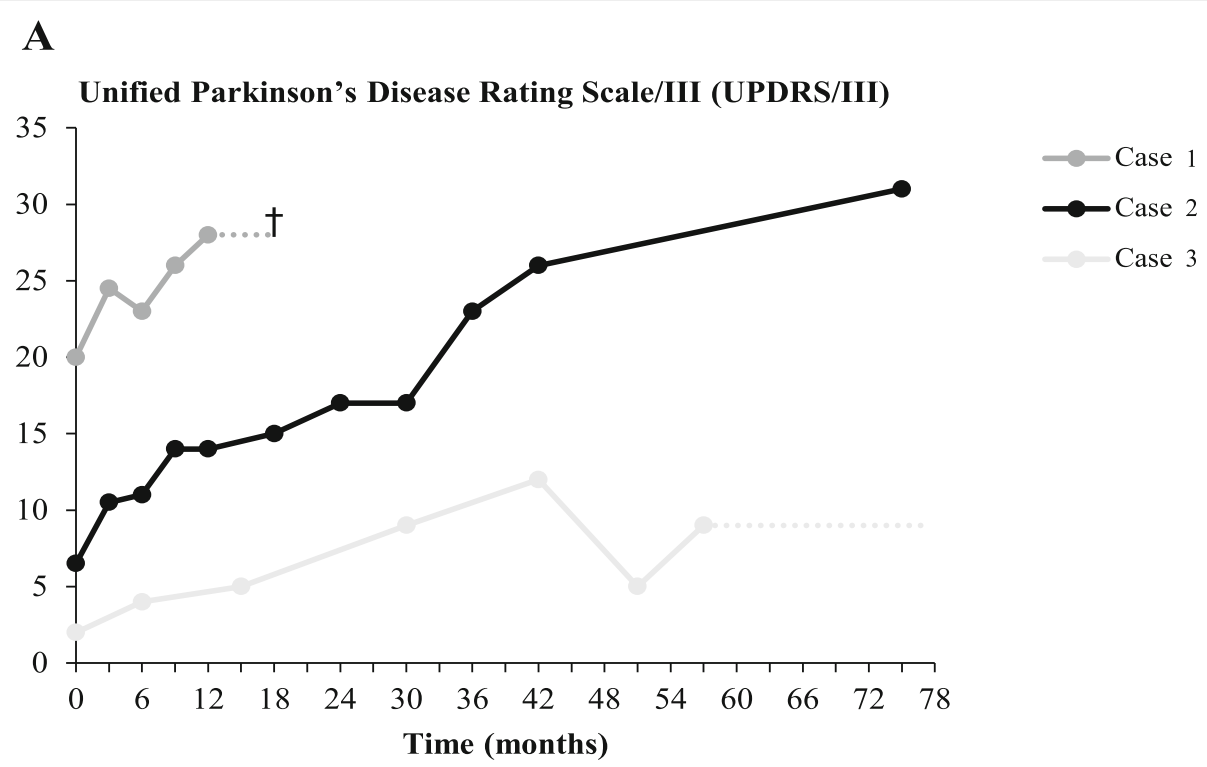

B

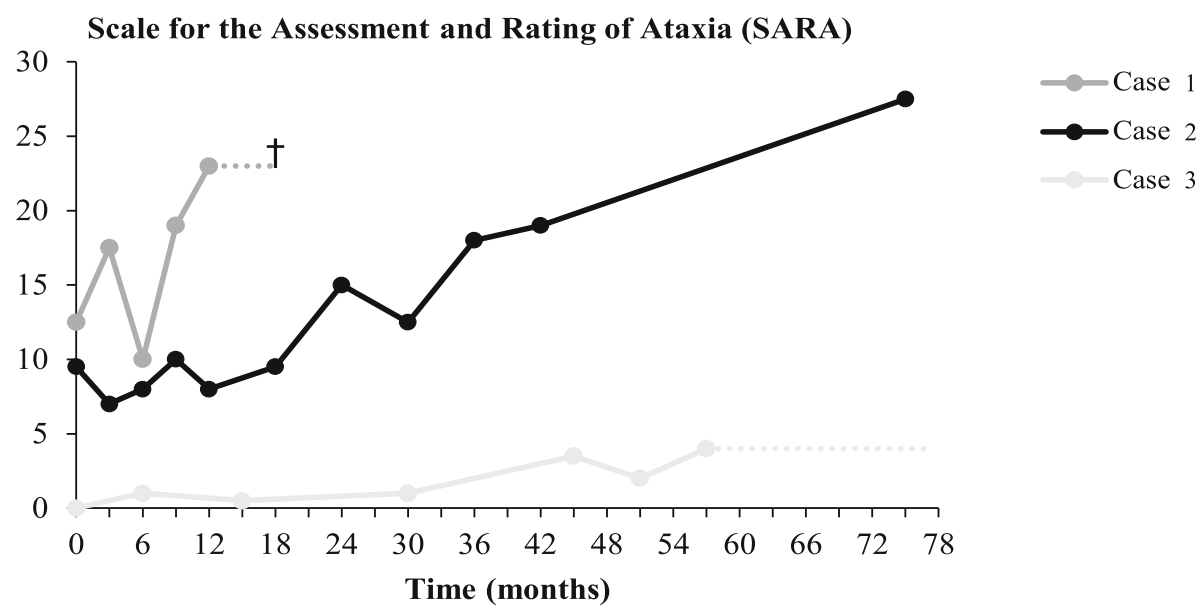

Fig. 1 Neurological rating scales of the G631R homozygous cases at baseline and during follow-up. Dotted lines indicate that motor function tests were no longer performed, which was due to neurological deterioration in case 1 and hospital relocation in case 3

(HADS) had only been consistently collected in case 1 and 2 , and were highly variable during treatment. Diabetes became manifest despite treatment in case 1 and 3 , while in case 2, who was already known with insulin-dependent diabetes at baseline, glucose and HbA1c levels improved and insulin doses could be reduced.

T2- and T2*-weighted MRI showed excessive iron accumulation in all cases at baseline, predominantly affecting basal ganglia, red nucleus, dentate nucleus and thalamus. Imaging was performed at $1.5 \mathrm{~T}$ in case 1 and $3 \mathrm{~T}$ in case 2 and 3. Qualitative follow-up revealed no significant changes in the pattern of iron accumulation nor in the severity of hypointensities in case 2 and 3, while in case 1 progressive darkening of deep grey matter regions was observed (Additional file 2).

\section{Review of the literature}

Therapeutic approaches for aceruloplasminemia have been described in 48 cases worldwide [7, 8, 15-49], including our three patients. Overall, 13 different iron chelating methods have been administered, of which a detailed overview is included in Additional file 3. Monotherapy with deferoxamine or deferasirox has been most frequently reported, while combined iron chelation therapy was limited to 5 previously reported cases $[8,17,40,44,48]$. In these patients, phlebotomy was combined with deferasirox, deferiprone with deferoxamine, and fresh frozen plasma with deferoxamine or deferiprone. In 7 others, an iron chelator was combined with zinc, vitamin $\mathrm{C}$ or $\mathrm{E}[17,30,33]$. The consecutive use of different chelators or 
combinations of iron chelating methods has been described in 10 patients [7, 16-19, 21, 22, 24, 27].

Table 2 summarizes the clinical characteristics of all 48 cases treated for aceruloplasminemia, differentiated between neurologically symptomatic patients $(n=24)$ and patients who started treatment before the onset of neurological manifestations $(n=24)$. These neurologically asymptomatic patients were diagnosed following the onset of diabetes and/or anemia [7, 17, 22, 28, 43, 47], during the diagnostic workup of unexplained hyperferritinemia with low transferrin saturation $[8,17,20,23,39,43,46,47]$, or by family counselling $[16,21,24,25,27,31,37,42]$.

Treatment in neurologically asymptomatic patients was started 11.5 years before neurological sequelae of aceruloplasminemia were likely to develop, based on the natural course of the disease observed in neurologically symptomatic patients $(p=0.003)$. However, the age at end of follow-up of initially asymptomatic patients was not significantly different from the age at onset of neurological manifestations in the symptomatic group $(p=$ 0.918 ), while only 9 of the 24 initially asymptomatic patients had developed neurological manifestations at that age. Kaplan-Meier analysis revealed that initiation of treatment in a presymptomatic stage of the disease might delay the onset of neurological manifestation by 10 years $(p=0.001$, Table 3 and Fig. 2).

Clinical outcomes of treatment in neurologically symptomatic patients were variable. Overall, neurological manifestations remained stable or improved in 11 patients, while 9 patients deteriorated neurologically despite treatment. In 4 cases, clinical outcomes of treatment were not available. Quantitative evaluations of neurological function were available in 8 of the 48 cases, using various clinical rating scales. More detailed information on the neurological outcomes of treatment in individual patients can be obtained from Additional file 3. It should be noted, though, that the median duration of treatment in patients who remained stable or improved neurologically was significantly shorter than patients in whom neurological deterioration was observed (6 months vs. 43 months, $p=0.016$ ). The limited available data on age at death precluded further analysis of any treatmentrelated influences on mortality in aceruloplasminemia.

\section{Discussion}

Data from published case reports and case series of aceruloplasminemia were combined to improve our understanding of the natural history of the disease and to systematically address the disease-modifying potential of iron chelation therapy. In addition, this is the first study that describes combined iron chelation therapy with deferiprone and phlebotomy for aceruloplasminemia.

Results of up to 3 years of combined iron chelation therapy with deferiprone and phlebotomy showed that the combination can be safely performed in aceruloplasminemia patients when treatment is subject to strict monitoring of hemoglobin and granulocyte count. This new therapeutic approach was initiated in three Dutch patients at a neurologically symptomatic stage of the disease. One patient, who was known with microcytic anemia, did not tolerate phlebotomy and was subsequently treated by deferiprone in combination with deferoxamine. Although neither deferiprone with phlebotomy nor deferiprone with deferoxamine could prevent further neurological deterioration, this report yields detailed information on clinical manifestations, adverse events associated with treatment and disease progression in various stages of the disease, which might be valuable for individual patient management.

The rate of neurological deterioration was markedly different between our patients. The slower progression of neurological manifestations in case 3, who had

Table 2 Clinical characteristics of patients with and without neurological manifestations when treatment was initiated

\begin{tabular}{llll}
\hline & Symptomatic $n=24$ & Asymptomatic $n=24$ & $p$ \\
\hline Male gender (\%) & $11(45.8)$ & $13(54.2)$ & $4(16.7)$ \\
Japanese ethnicity (\%) & $8(33.3)$ & & 0.564 \\
Natural history & & $36.5(20-43)$ & \\
Age at first manifestations, $y$ & $35(26-49)$ & - & 0.443 \\
Age at first neurological manifestations, $y$ & $51(48.5-55)$ & $39.5(35-49)$ & - \\
Treatment and clinical outcomes & & $72(24-120)$ & 0.001 \\
Age at start of treatment, y & $55(50.5-59)$ & $53(47-61)$ & 0.001 \\
Duration of treatment, $m$ & $14(5.5-48)$ & $50(45-55)$ & $n=9$ \\
Age at end of follow-up, $y$ & $58(54-63)$ & 67 & $n=1$ \\
Age at first neurological manifestations, $y$ & - & $n=8$ & - \\
Age at death, $y$ & $60(56-65.5)$ & & $n$ \\
\hline
\end{tabular}

Abbreviations: $n$ number, $y$ years, $m$ months

Ages and duration of treatment are expressed as median (interquartile range) 
Table 3 Onset of neurological manifestations stratified by timing of treatment initiation

\begin{tabular}{lllll}
\hline Group & $\begin{array}{l}\text { Median age at } \\
\text { first neurological } \\
\text { manifestations } \\
\text { (years) }\end{array}$ & SE & $95 \% \mathrm{Cl}$ & $p$ \\
\hline $\begin{array}{l}\text { Symptomatic at } \\
\text { start treatment }\end{array}$ & 51 & 0.6 & $49.8-52.2$ & 0.001 \\
$\begin{array}{l}\text { Asymptomatic at } \\
\text { start treatment }\end{array}$ & 61 & 5.0 & $51.2-70.8$ & \\
\hline
\end{tabular}

minimal neurological complaints at baseline, might emphasize the importance of early initiation of iron chelation therapy to provide clinical benefit. The analysis of aggregated case reports of aceruloplasminemia supports this interpretation, as the estimated onset of neurological manifestations could be postponed by 10 years when treatment was initiated in a presymptomatic stage of the disease. In a recently published randomized controlled trial in patients with pantothenate kinase-associated neurodegeneration (PKAN), the most common form of NBIA, deferiprone seemed to have more potential to slow disease progression when it was initiated in patients with less advanced stages of neurodegeneration [50]. As such analyses of the rate of neurological progression and disease-modifying potential of treatment require quantitative evaluations of neurological function, accepted clinical rating scores should ideally be included in future case studies of aceruloplasminemia.

One of the major pitfalls of using case reports and case series is publication bias - cases with a positive response to treatment are much more likely to be published than those without clinical benefit [51, 52]. This could account for the discrepancy between the progression of neurological manifestations in our patients and the relatively high proportion of published cases that showed improvement of neurological sequelae during follow-up. The majority of these neurologically symptomatic cases that improved were followed over months, while results of those with long-term follow-up illustrated the debilitating nature of aceruloplasminemia and were in accordance with observations in our patients.

Treatment outcomes in neurologically asymptomatic patients are subject to specific types of information bias: lead-time bias and length-time bias. Lead-time bias indicates the overestimation of survival duration due to detection of disease in an early stage, while length-time bias addresses the systematic error resulting from a relative excess of cases with a slower rate of disease progression [53-55]. Although both types of information bias have been predominantly discussed in relation to cancer screening, lead-time bias should be considered when interpreting the sustained absence of neurological manifestations in reported aceruloplasminemia patients in whom treatment was initiated years before neurological manifestations were to expect. On the other hand, nearly half of the patients in whom treatment was initiated in a presymptomatic stage of the disease developed neurological manifestations above 55 years of age or remained asymptomatic despite being in their late fifties or sixties. Length-time bias might have additionally contributed to the more favourable neurological course of the disease in initially asymptomatic patients. However, the

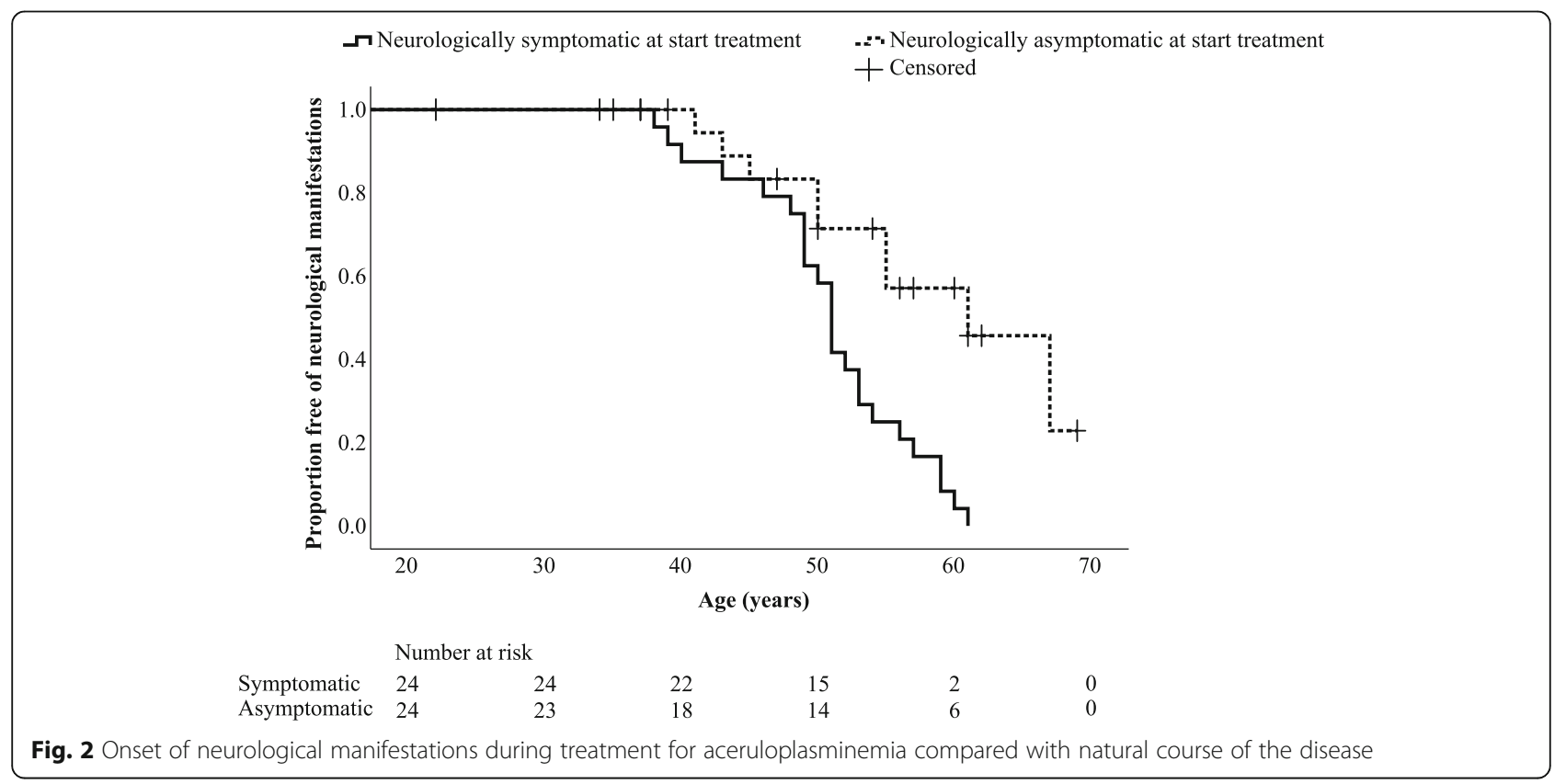


comparable age at first non-neurological manifestations of aceruloplasminemia in both neurologically symptomatic and asymptomatic patients weakens this consideration. In addition, no clear genotype-phenotype correlations have been reported in aceruloplasminemia [16]. Recent observations that suggest a milder phenotypic expression of mutations that allow the synthesis of apoceruloplasmin with some residual ferroxidase activity, need validation in larger series [17].

\section{Conclusions}

Neurological outcomes of treatment for aceruloplasminemia, in both our three cases and other published cases, support the potential of iron chelation therapy to slow neurological progression when treatment is initiated in an early stage of the disease. Our analysis of aggregated case reports, which is the first of its kind in aceruloplasminemia, further highlights that reported outcomes of treatment in individual cases should be cautiously used to select the optimal therapeutic approach for aceruloplasminemia, given the possibly misleading contribution of various types of bias and often unsatisfying duration of follow-up. It underlines the need for further prospective studies using agreed treatment regimen with long-term follow-up. Based on the observed longterm safety and tolerability in our study and its theoretical superiority, combined iron chelation therapy with deferiprone and phlebotomy is recommended for patients without symptomatic anemia. In the presence of symptomatic anemia and decreased tolerability of phlebotomy, deferiprone with deferoxamine or deferiprone combined with another iron chelating method might be a reasonable alternative approach to achieve efficient iron chelation in aceruloplasminemia.

\section{Supplementary information}

Supplementary information accompanies this paper at https://doi.org/10. 1186/s13023-020-01385-W

Additional file 1. Detailed overview of biochemical parameters of the G631R homozygous cases at baseline and during follow-up.

Additional file 2. Qualitative assessment of brain iron by T2-weighted $\mathrm{MRI}$ at $1.5 \mathrm{~T}$ in case 1.

Additional file 3. Detailed overview of published treatments for aceruloplasminemia.

\section{Abbreviations}

CP: Ceruloplasmin; HADS: Hospital Anxiety and Depression Scale; NBIA: Neurodegeneration with brain iron accumulation; PKAN: Pantothenate kinase-associated neurodegeneration; SARA: Scale for the Assessment and Rating of Ataxia; UPDRS: Unified Parkinson's Disease Rating Scale

\section{Acknowledgements}

We are grateful to Adam Doyle, Alfonso Fasano, Frank Lammert, Uwe Lindner, Olivier Loréal, Loris Poli, Monica Rusticeanu, Ulrich Stölzel, Toshikazu Yoshida, Kunuhiro Yoshida and Vincent Zimmer for responding to our e-mail and providing additional follow-up.

\section{Authors' contributions}

L.V. collected data, wrote the manuscript and constructed figures and tables. A.B. designed the study, collected data with neurological examinations, reviewed and edited the manuscript. P.W. designed the study, reviewed and edited the manuscript. J.L. initiated the study and formulated the research question, collected data, reviewed and edited the manuscript. The author(s) read and approved the final manuscript.

\section{Funding}

This research received no specific grant from any funding agency in the public, commercial or not-for-profit sectors.

\section{Availability of data and materials}

All data generated or analysed during this study are included in the published article and its supplementary information files.

Ethics approval and consent to participate

This study was approved by the Medical Ethics Review Board Erasmus MC (approval number: MEC-2013-280)

\section{Consent for publication}

Participants provided written informed consent.

\section{Competing interests}

The authors declare that they have no competing interests.

\section{Author details}

'Department of Internal Medicine, Center for Lysosomal and Metabolic Diseases, Porphyria Center Rotterdam, Erasmus MC University Medical Center Rotterdam, Rotterdam, The Netherlands. ${ }^{2}$ Department of Neurology, Erasmus MC University Medical Center, Rotterdam, The Netherlands.

Received: 28 January 2020 Accepted: 14 April 2020

Published online: 25 April 2020

\section{References}

1. Miyajima H. Aceruloplasminemia. Neuropathology. 2015;35:83-90.

2. Piperno A, Alessio M. Aceruloplasminemia: waiting for an efficient therapy Front Neurosci. 2018;12:903.

3. Dusek P, Schneider SA, Aaseth J. Iron chelation in the treatment of neurodegenerative diseases. J Trace Elem Med Biol. 2016;38:81-92.

4. Patel BN, David S. A novel glycosylphosphatidylinositol-anchored form of ceruloplasmin is expressed by mammalian astrocytes. J Biol Chem. 1997;272: 20185-90.

5. Jeong SY, David S. Glycosylphosphatidylinositol-anchored ceruloplasmin is required for iron efflux from cells in the central nervous system. J Biol Chem. 2003;278:27144-8.

6. Evans RW, Sharma M, Ogwand W, Patel KJ, Bartlett AN, Kontoghiorghes GJ. The effect of alpha-ketohydroxypyridine chelators on transferrin saturation in vitro and in vivo. Drugs Today. 1992;28:19-23.

7. Mariani R, Arosio C, Pelucchi S, Grisoli M, Piga A, Trombini P, et al. Iron chelation therapy in aceruloplasminaemia: study of a patient with a novel missense mutation. Gut. 2004;53:756-8.

8. Badat M, Kaya B, Telfer P. Combination-therapy with concurrent deferoxamine and deferiprone is effective in treating resistant cardiac ironloading in aceruloplasminaemia. Br J Haematol. 2015;171:430-2.

9. Vlachodimitropoulou Koumoutsea E, Garbowski M, Porter J. Synergistic intracellular iron chelation combinations: mechanisms and conditions for optimizing iron mobilization. Br J Haematol. 2015;170:874-83.

10. Hider RC, Hoffbrand AV. The role of Deferiprone in Iron chelation. $\mathrm{N}$ Engl J Med. 2018;379:2140-50.

11. Sampayo-Cordero M, Miguel-Huguet B, Pardo-Mateos A, Malfettone A, Perez-Garcia J, Llombart-Cussac A, et al. Agreement between results of meta-analyses from case reports and clinical studies, regarding efficacy and safety of idursulfase therapy in patients with mucopolysaccharidosis type II (MPS-II). A new tool for evidence-based medicine in rare diseases. Orphanet J Rare Dis. 2019;14:230

12. Sampayo-Cordero M, Miguel-Huguet B, Pardo-Mateos A, Molto-Abad M, Munoz-Delgado C, Perez-Lopez J. Agreement between the results of metaanalyses from case reports and from clinical studies regarding the efficacy of laronidase therapy in patients with mucopolysaccharidosis type I who 
initiated enzyme replacement therapy in adult age: an example of case reports meta-analyses as an useful tool for evidence-based medicine in rare diseases. Mol Genet Metab. 2018;123:69-75.

13. Vroegindeweij $L H$, van der Beek EH, Boon AJ, Hoogendoorn M, Kievit JA, Wilson $\mathrm{JH}$, et al. Aceruloplasminemia presents as type 1 diabetes in nonobese adults: a detailed case series. Diabet Med. 2015;32:993-1000.

14. Hellman NE, Kono S, Mancini GM, Hoogeboom AJ, De Jong GJ, Gitlin JD. Mechanisms of copper incorporation into human ceruloplasmin. J Biol Chem. 2002:277:46632-8.

15. Kerkhof M, Honkoop P. Never forget aceruloplasminemia in case of highly suggestive Wilson's disease score. Hepatology. 2014;59:1645-7.

16. Vroegindeweij $L H$, Langendonk JG, Langeveld $M$, Hoogendoorn $M$, Kievit AJA, Di Raimondo D, et al. New insights in the neurological phenotype of aceruloplasminemia in Caucasian patients. Parkinsonism Relat Disord. 2017 36:33-40.

17. Pelucchi S, Mariani R, Ravasi G, Pelloni I, Marano M, Tremolizzo L, et al. Phenotypic heterogeneity in seven Italian cases of aceruloplasminemia. Parkinsonism Relat Disord. 2018;51:36-42.

18. Pelucchi S, Pelloni I, Arosio C, Mariani R, Piperno A. Does aceruloplasminemia modulate iron phenotype in thalassemia intermedia? Blood Cells Mol Dis. 2016:57:112-4.

19. Bethlehem C, van Harten B, Hoogendoorn M. Central nervous system involvement in a rare genetic iron overload disorder. Neth J Med. 2010;68: 316-8.

20. Bjork MH, Gjerde IO, Tzoulis C, Ulvik RJ, Bindoff LA. A man in his 50s with high ferritin levels and increasing cognitive impairment. Tidsskr Nor Laegeforen. 2015;135:1369-72.

21. Bove F, Fasano A. Iron chelation therapy to prevent the manifestations of aceruloplasminemia. Neurology. 2015:85:1085-6.

22. Calder GL, Lee MH, Sachithanandan N, Bell S, Zeimer H, Maclsaac RJ. Aceruloplasminaemia: a disorder of diabetes and neurodegeneration. Intern Med J. 2017:47:115-8.

23. Doyle A, Rusli F, Bhathal P. Aceruloplasminaemia: a rare but important cause of iron overload. BMJ Case Rep. 2015:bcr2014207541. https://doi.org/10. 1136/bcr-2014-207541

24. Fasano A, Colosimo C, Miyajima H, Tonali PA, Re TJ, Bentivoglio AR. Aceruloplasminemia: a novel mutation in a family with marked phenotypic variability. Mov Disord. 2008;23:751-5.

25. Finkenstedt A, Wolf E, Hofner E, Gasser BI, Bosch S, Bakry R, et al. Hepatic but not brain iron is rapidly chelated by deferasirox in aceruloplasminemia due to a novel gene mutation. J Hepatol. 2010;53:1101-7.

26. Haemers I, Kono S, Goldman S, Gitlin JD, Pandolfo M. Clinical, molecular, and PET study of a case of aceruloplasminaemia presenting with focal cranial dyskinesia. J Neurol Neurosurg Psychiatry. 2004;75:334-7.

27. Hayashida M, Hashioka S, Miki H, Nagahama M, Wake R, Miyaoka T, et al. Aceruloplasminemia with psychomotor excitement and neurological sign was improved by minocycline (case report). Medicine (Baltimore). 2016;95: e3594.

28. Hellman NE, Schaefer M, Gehrke S, Stegen P, Hoffman WJ, Gitlin JD, et al. Hepatic iron overload in aceruloplasminaemia. Gut. 2000;47:858-60.

29. Hida A, Kowa H, Iwata A, Tanaka M, Kwak S, Tsuji S. Aceruloplasminemia in a Japanese woman with a novel mutation of CP gene: clinical presentations and analysis of genetic and molecular pathogenesis. J Neurol Sci. 2010;298: 136-9.

30. Hines MC Jr, Bonkovsky HL, Rudnick SR, Mhoon JT. Peripheral neuropathy and the Ceruloplasmin gene. Ann Intern Med. 2018;168:894-5.

31. Lindner U, Schuppan D, Schleithoff L, Habeck JO, Grodde T, Kirchhof K, et al. Aceruloplasminaemia: a family with a novel mutation and long-term therapy with deferasirox. Horm Metab Res. 2015;47:303-8.

32. Logan Jl, Harveyson KB, Wisdom GB, Hughes AE, Archbold GP. Hereditary caeruloplasmin deficiency, dementia and diabetes mellitus. Qjm. 1994:87: 663-70.

33. Loreal O, Turlin B, Pigeon C, Moisan A, Ropert M, Morice $\mathrm{P}$, et al. Aceruloplasminemia: new clinical, pathophysiological and therapeutic insights. J Hepatol. 2002;36:851-6.

34. Matsushima A, Yoshida T, Yoshida K, Ohara S, Toyoshima Y, Kakita A, et al. Superficial siderosis associated with aceruloplasminemia. Case report. J Neurol Sci. 2014:339:231-4

35. Miyajima H, Nishimura Y, Mizoguchi K, Sakamoto M, Shimizu T, Honda N. Familial apoceruloplasmin deficiency associated with blepharospasm and retinal degeneration. Neurology. 1987;37:761-7.
36. Miyajima H, Takahashi Y, Kamata T, Shimizu H, Sakai N, Gitlin JD. Use of desferrioxamine in the treatment of aceruloplasminemia. Ann Neurol. 1997; 41:404-7.

37. Ogimoto M, Anzai K, Takenoshita H, Kogawa K, Akehi Y, Yoshida R, et al. Criteria for early identification of aceruloplasminemia. Intern Med. 2011;50: 1415-8.

38. Pan PL, Tang HH, Chen Q, Song W, Shang HF. Desferrioxamine treatment of aceruloplasminemia: long-term follow-up. Mov Disord. 2011;26:2142-4.

39. Perez-Aguilar F, Burguera JA, Benlloch S, Berenguer M, Rayon JM. Aceruloplasminemia in an asymptomatic patient with a new mutation. Diagnosis and family genetic analysis. J Hepatol. 2005:42:947-9.

40. Poli L, Alberici A, Buzzi P, Marchina E, Lanari A, Arosio C, et al. Is aceruloplasminemia treatable? Combining iron chelation and fresh-frozen plasma treatment. Neurol Sci. 2017;38:357-60.

41. Riboldi GM, Anstett K, Jain R, Lau H, Swope D. Aceruloplasminemia and putaminal cavitation. Parkinsonism Relat Disord. 2018;51:121-3.

42. Roberti Mdo R, Borges Filho HM, Goncalves CH, Lima FL. Aceruloplasminemia: a rare disease - diagnosis and treatment of two cases. Rev Bras Hematol Hemoter. 2011;33:389-92.

43. Rusticeanu M, Zimmer V, Schleithoff L, Wonney K, Viera J, Zimmer A, et al. Novel ceruloplasmin mutation causing aceruloplasminemia with hepatic iron overload and diabetes without neurological symptoms. Clin Genet. 2014;85:300-1.

44. Skidmore FM, Drago V, Foster P, Schmalfuss IM, Heilman KM, Streiff RR. Aceruloplasminaemia with progressive atrophy without brain iron overload: treatment with oral chelation. J Neurol Neurosurg Psychiatry. 2008:79:46770 .

45. Suzuki Y, Yoshida K, Aburakawa Y, Kuroda K, Kimura T, Terada T, et al. Effectiveness of oral iron chelator treatment with deferasirox in an aceruloplasminemia patient with a novel ceruloplasmin gene mutation. Intern Med. 2013:52:1527-30.

46. Tai M, Matsuhashi N, Ichii O, Suzuki T, Ejiri Y, Kono S, et al. Case of presymptomatic aceruloplasminemia treated with deferasirox. Hepatol Res. 2014:44:1253-8

47. Watanabe M, Ohyama K, Suzuki M, Nosaki Y, Hara T, Iwai K, et al. Aceruloplasminemia with abnormal compound heterozygous mutations developed neurological dysfunction during phlebotomy therapy. Intern Med. 2018;57:2713-8.

48. Yonekawa M, Okabe T, Asamoto Y, Ohta M. A case of hereditary ceruloplasmin deficiency with iron deposition in the brain associated with chorea, dementia, diabetes mellitus and retinal pigmentation: administration of fresh-frozen human plasma. Eur Neurol. 1999:42:157-62.

49. Morita H, Ikeda S, Yamamoto K, Morita S, Yoshida K, Nomoto S, et al. Hereditary ceruloplasmin deficiency with hemosiderosis: a clinicopathological study of a Japanese family. Ann Neurol. 1995;37:646-56.

50. Klopstock T, Tricta F, Neumayr L, Karin I, Zorzi G, Fradette C, et al. Safety and efficacy of deferiprone for pantothenate kinase-associated neurodegeneration: a randomised, double-blind, controlled trial and an open-label extension study. Lancet Neurol. 2019;18:631-42.

51. Murad MH, Sultan S, Haffar S, Bazerbachi F. Methodological quality and synthesis of case series and case reports. BMJ Evid Based Med. 2018;23:60-3.

52. Nissen T, Wynn R. The clinical case report: a review of its merits and limitations. BMC Res Notes. 2014;7:264.

53. Cucchetti A, Trevisani F, Pecorelli A, Erroi V, Farinati F, Ciccarese F, et al. Estimation of lead-time bias and its impact on the outcome of surveillance for the early diagnosis of hepatocellular carcinoma. J Hepatol. 2014;61:33341.

54. Zakko L, Visrodia K, Wang KK, lyer PG. Editorial: the effect of Bias on estimation of improved survival after diagnosis of Barrett's esophagus. Am J Gastroenterol. 2017;112:1265-6.

55. Duffy SW, Nagtegaal ID, Wallis M, Cafferty FH, Houssami N, Warwick J, et al. Correcting for lead time and length bias in estimating the effect of screen detection on cancer survival. Am J Epidemiol. 2008;168:98-104.

\section{Publisher's Note}

Springer Nature remains neutral with regard to jurisdictional claims in published maps and institutional affiliations. 